19 Stein PD, Henry JW. Prevalence of acute pulmonary embolism among patients in a general hospital and at autopsy. Chest 1995; 108:978-81.

20 Engelke C, Rummeny EJ, Marten K. Pulmonary embolism at multi-detector row CT of chest: oneyear survival of treated and untreated patients. Radiology 2006;239:563-75.
21 Le Gal G, Righini M, Parent F, et al. Diagnosis and management of subsegmental pulmonary embolism. J Thromb Haemost 2006:4:724-31.

22 Gurney JW. No fooling around: direct visualisation of pulmonary embolism. Radiology 1993; 188:618-9.

23 Prologo JD, Gilkeson RC, Diaz M, et al. The effect of single-detector CT versus MDCT on clinical outcomes in patients with suspected acute pulmonary embolism and negative results on $\mathrm{CT}$ pulmonary angiography. AJR Am J Roentgenol 2005;184:1231-5.

24 Iles S. Clot burden and comorbidity in natural history of untreated pulmonary thromboembolism: autopsy data in the trial by Barritt and Jordan. Chest 2003;124:1178-9.

\title{
Acute renal failure in people with cystic fibrosis
}

\section{Kevin W Southern}

\section{Time to reflect on antibiotic strategies for CF lung infection}

$\mathrm{T}$ he putative gene in cystic fibrosis (CF) encodes a protein, cystic fibrosis transmembrane conductance regulator (CFTR), which has an important role in transepithelial salt transport. ${ }^{1}$ The major organ affected in CF is the lung, with remorseless and intense chronic airway infection resulting from disabled clearance of dehydrated airway surface liquid. ${ }^{2}$ Airway inflammation leading to end stage lung damage is associated with respiratory morbidity and early death. The outlook for people with CF has improved considerably with a proactive approach to treatment of airway infection as one of the cornerstones of its management. Recent data from the US CF registry suggest a continuing improvement in median predicted survival to over 35 years (http://www.cff.org/research/ 2006NACFC/Plenary III). With an improving outlook, perspectives have changed and expectations of people with CF and their carers have broadened. A survey of acute renal failure (ARF) in patients with CF published in this issue of Thorax (see $p$ 541) is particularly timely when reflecting on how best to achieve the goal of increased survival while balancing risks to patients. ${ }^{3}$

\section{ACUTE RENAL FAILURE AND CF}

There are several reasons why people with $\mathrm{CF}$ are at risk of ARF. The salt transport defect makes people with CF prone to salt loss and consequently fluid imbalance and dehydration. ${ }^{45}$ The impact of CFTR dysfunction on pancreatic function and architecture results in significant hypoinsulinaemia (CF-related diabetes) in a significant proportion of older patients with $\mathrm{CF}$, again posing a challenge to fluid balance and the long-term possibility of diabetic kidney disease. ${ }^{6}$ Finally, as a cohort, these patients are exposed to industrial quantities of potentially nephrotoxic chemotherapeutic agents (in our CF centre some children have received a cumulative intravenous dose of over $300 \mathrm{~g}$ of the aminoglycoside tobramycin). Given cumulative exposure to potentially nephrotoxic drugs and the increased prevalence of CF-related diabetes in older people with $\mathrm{CF}$, an increased incidence risk of ARF might be expected in the older age group; however, this was not the case in this survey where the majority of cases were children (only three over 18 years). This may represent better response rates from paediatric centres to this survey, and further studies are needed to examine this finding.

\section{ROLE OF CFTR IN THE KIDNEY}

Paradoxically for a condition in which salt transport is a primary abnormality, people with CF have apparently normal renal function. The CFTR gene is expressed abundantly in the kidney, particularly in the nephron, but CFTR appears to be functionally redundant at this site. ${ }^{8}$ Studies on a transgenic cftr knockout mouse model suggest that the absence of CFTR does not affect the ability of the kidney to manage fluid and salt imbalance, but that different salt and fluid transport processes are involved. ${ }^{9} \quad$ Interestingly, CFTR does appear to have a role in the pathophysiology of autosomal recessive polycystic kidney disease, where fluid secretion into the cysts appears to be mediated through CFTR. ${ }^{10}{ }^{11}$ The increased incidence of nephrocalcinosis in CF does not appear to relate to an intrinsic renal problem and is more likely related to hyperoxaluria. $^{12}$

Given the apparent pivotal role of CFTR in other organs, the lack of impact of CFTR dysfunction on the kidney is remarkable. It is even possible that absent CFTR function in the kidney may protect people with CF from renal insults, and certainly it has been recognised for many years that people with CF have increased renal clearance of many drugs including aminoglycoside antibiotics. ${ }^{13}$ More research is needed into the $\mathrm{CF}$ renal condition to understand the impact of CFTR dysfunction on renal physiology.

\section{RELEVANCE OF ACUTE RENAL FAILURE IN CF}

The incidence risk of ARF reported by Bertenshaw et al $^{3}$ is significantly higher than the non-CF population and is consistent with other recent reports. ${ }^{15}$ The authors suggest this may be an increasing phenomenon, quoting local figures, although there are no previous national data to assess this. Because of problems with recruitment and case verification, it is likely that the incidence risk quoted is an underestimate. It is important that CF teams reflect on these figures and, as a first line, adopt "common sense" procedures to prevent inadvertent renal damage. Patients should be monitored more closely during periods of hot weather and during fasts, particularly if using aminoglycoside antibiotics (for example, to improve a chest condition before a general anaesthesia). In addition, patients should avoid the concomitant use of potentially nephrotoxic agents such as ibuprofen, frusemide and aminoglycosides. If concomitant use is unavoidable, then careful monitoring is imperative.

Given the increased incidence of ARF in $\mathrm{CF}$, there is a strong argument for more formal assessment of renal function on a regular basis in order to highlight at risk individuals. The glomerular filtration rate can be estimated by formulae that employ serum creatinine and other parameters such as height, weight and age. The Schwarz formula $\left(40^{*}\right.$ height $(\mathrm{cm}) /$ serum creatinine $(\mu \mathrm{mol} / \mathrm{l}))$ is commonly used; however, many formulae exist but none has been formally validated in $\mathrm{CF} .{ }^{16} \mathrm{~A}$ concern is that these formulae 
may overestimate the glomerular filtration rate in people with $\mathrm{CF}$ and more formal direct measurement may be required for individuals who are particularly at risk (for example, using ${ }^{51} \mathrm{Cr}$ EDTA or timed urine collections). ${ }^{13}$

\section{SHOULD WE ALTER/REDUCE OUR USE OF AMINOGLYCOSIDES?}

Aminoglycosides provide excellent dosedependent killing of Pseudomonas aeruginosa, which remains the primary pathogen in CF. ${ }^{17}$ They act synergistically with a number of classes of antibiotics including cephalosporins, and have been used in combination in an attempt to prevent the emergence of resistance. A systematic review comparing single and combination intravenous antibiotic strategies could only identify weak evidence to support this strategy. ${ }^{18}$ This highlights the challenge of evaluating treatments for life long conditions with the short-term outcomes that are generally available to systematic reviews. ${ }^{19}$ Despite a dearth of evidence, the increasing resistance of $P$ aeruginosa and the emergence of epidemic strains make it likely that aminoglycosides will continue to have a role in the CF armamentarium. However, innovative approaches to their use should be considered.

Combining aerosolised aminoglycosides with an intravenous cephalosporin could decrease the nephrotoxic potential while maintaining some protection against the development of resistance. A pilot study of this approach suggested equivalent efficacy. ${ }^{20}$ However, ARF with aerosolised tobramycin in isolation has been reported and renal function would still need to be monitored carefully. ${ }^{21}$

Rotation of antibiotic regimes may reduce exposure to aminoglycosides. Intravenous colistin (a polymixin) has been advocated as an alternative to aminoglycosides and, in a survey of adult patients, was not associated with renal compromise unless given in combination with tobramycin. ${ }^{15}$ Many CF centres give routine 3- or 4-monthly intravenous antibiotics to patients with chronic $P$ aeruginosa infection. Again this may be a policy that $\mathrm{CF}$ teams should review in light of the cumulative exposure of potentially nephrotoxic agents to patients and the lack of an evidence base.22

Sixteen of the 24 cases reported in the survey by Bertenshaw et al received gentamicin. ${ }^{3}$ The study group are embarking on a more rigorous case-control study to investigate factors impacting on the development of ARF; however, with the data presented in this report, the use of intravenous gentamicin in the routine management of patients with CF cannot be supported. Alternative options should be employed and the infrastructure established to support this with appropriate drug assays.

\section{ONCE DAILY DOSING OF TOBRAMYCIN}

Tobramycin, which has a lower nephrotoxic potential than gentamicin, is the most widely used intravenous aminoglycoside antibiotic in the UK for CF chest disease. At present a large number of CF units are changing to a once daily dosage regime. Once daily dosing reduces drug accumulation and potentially reduces the risk of nephrotoxicity and ototoxicity. Fears of a reduction in efficacy because of the prompt renal clearance in CF (and increased time below the minimum inhibitory concentration) have been allayed by the TOPIC study. ${ }^{23}$ This randomised controlled trial was sufficiently powered to demonstrate equivalent efficacy between once and thrice daily regimes, with no significant toxicity. A subsequent inadequately powered trial has raised the concern that once daily dosing may increase the resistance profile of $P$ aeruginosa. ${ }^{24}$ Moving to a once daily dosing regime is a significant change in practice and CF teams need to ensure robust protocols are in place and that dosing regimes are correct. There is still debate as to the most appropriate scheme to monitor once daily dosing. Whether there is a need to measure serum levels and renal function will need to be clarified. ${ }^{25}$ The overall impact of this change in practice on the incidence risk of ARF requires careful evaluation.

In summary, Bertenshaw and colleagues have provided us with valuable insight into an uncommon but catastrophic complication of $\mathrm{CF}^{3}$ In light of these findings, CF teams should reflect on their management strategies, particularly with regard to the use of aminoglycoside antibiotics. Further work is required to clarify the renal condition in CF so that we can better understand long-term risks to people with CF and develop validated tools to monitor renal function in this population.

Thorax 2007;62:472-473.

doi: $10.1136 /$ thx.2006.072355

Correspondence to: Dr Kevin W Southern Institute of Child Health, University of Liverpool, Royal Liverpool Children's Hospital, Liverpool L12 2AP, UK; kwsouth@liv.ac.uk

Competing interests: None

\section{REFERENCES}

1 Riordan JR, Rommens JM, Kerem B, et al. Identification of the cystic fibrosis gene: cloning and characterization of complementary DNA. Science 1989;245: 1066-73.

2 Matsui H, Wagner VE, Hill DB, et al. A physica linkage between cystic fibrosis airway surface dehydration and Pseudomonas aeruginosa biofilms. Proc Natl Acad Sci USA 2006;103:18131-6

3 Bertenshaw C, Watson AR, Lewis S, et al. Survey of acute renal failure in patients with cystic fibrosis in the UK. Thorax 2007;62:541-5.

4 Ballestero Y, Hernandez Ml, Rojo P, et al. Hyponatremic dehydration as a presentation of cystic fibrosis. Pediatr Emerg Care 2006;22:725-7.

5 Kriemler S, Wilk B, Schurer W, et al. Preventing dehydration in children with cystic fibrosis who exercise in the heat. Med Sci Sports Exerc 1999:31:774-9.

6 Brennan AL, Geddes DM, Gyi KM, et al. Clinical importance of cystic fibrosis-related diabetes. J Cyst Fibros 2004;3:209-22.

7 Quattrucci S, Rolla M, Cimino G, et al. Lung transplantation for cystic fibrosis: 6-year follow-up. J Cyst Fibros 2005;4:107-14.

8 Stanton BA. Cystic fibrosis transmembrane conductance regulator (CFTR) and renal function. Wien Klin Wochenschr 1997:109:457-64.

9 Kibble JD, Balloch KJ, Neal AM, et al. Rena proximal tubule function is preserved in Cftr $(\mathrm{tm} 2$ cam) deltaF508 cystic fibrosis mice. J Physiol 2001;532:449-57.

10 Nakanishi K, Sweeney WE Jr, Macrae Dell K, et al. Role of CFTR in autosomal recessive polycystic kidney disease. J Am Soc Nephrol $2001 ; 12: 719-25$

11 Xu N, Glockner JF, Rossetti S, et al. Autosomal dominant polycystic kidney disease coexisting with cystic fibrosis. J Nephrol 2006; 19:529-34.

12 Hoppe B, von Unruh GE, Blank G, et al. Absorptive hyperoxaluria leads to an increased risk for urolithiasis or nephrocalcinosis in cystic fibrosis. Am J Kidney Dis 2005;46:440-5.

13 Assael BM, Marra G, Tirelli AS, et al. Renal function in cystic fibrosis. Int J Pediatr Nephrol 1986:7:213-6.

14 Touw DJ. Clinical pharmacokinetics of antimicrobial drugs in cystic fibrosis. Pharm World Sci 1998;20:149-60.

15 Al-Aloul M, Miller H, Alapati S, et al. Renal impairment in cystic fibrosis patients due to repeated intravenous aminoglycoside use. Pediatr Pulmonol 2005;39:15-20.

16 Al-Aloul M, Jackson M, Bell G, et al. Comparison of methods of assessment of renal function in cystic fibrosis (CF) patients. J Cyst Fibros 2007;6:41-7.

17 Jana S, Deb JK. Molecular understanding of aminoglycoside action and resistance. Appl Microbiol Biotechnol 2006:70:140-50.

18 Elphick HE, Tan A. Single versus combination intravenous antibiotic therapy for people with cystic fibrosis. Cochrane Database Syst Rev 2005;(2):CD002007.

19 Elphick HE, Tan A, Ashby D, et al. Systematic reviews and lifelong diseases. $B M J$ 2002;325:381-4.

20 Al-Aloul M. A randomised cross over trial of TOB versus intravenous tobramycin in acute pulmonary exacerbations in CF. Pediatr Pulmonol 2004; (Supp 27): 249 .

21 Hoffmann IM, Rubin BK, Iskandar SS, et al. Acute renal failure in cystic fibrosis: association with inhaled tobramycin therapy. Pediatr Pulmonol 2002;34:375-7.

22 Elborn JS, Prescott RJ, Stack BH, et al. Elective versus symptomatic antibiotic treatment in cystic fibrosis patients with chronic Pseudomonas infection of the lungs. Thorax 2000;55:355-8.

23 Smyth A, Tan KH, Hyman-Taylor P, et al. Once versus three-times daily regimens of tobramycin treatment for pulmonary exacerbations of cystic fibrosis - the TOPIC study: a randomised controlled trial. Lancet 2005;365:573-8.

24 Burkhardt O, Lehmann C, Madabushi R, et al. Once-daily tobramycin in cystic fibrosis: better for clinical outcome than thrice-daily tobramycin but more resistance development? J Antimicrob Chemother 2006;58:822-9.

25 Tan KH, Mulheran M, Knox AJ, et al Aminoglycoside prescribing and surveillance in cystic fibrosis. Am J Respir Crit Care Med 2003;167:819-23. 